\title{
Pazopanib in patients with metastatic renal cell carcinoma: a single-center, real-world, retrospective Chinese study
}

\author{
Jianhui Chen ${ }^{1} \wedge$, Wen Ye ${ }^{1}$, Wei Jiang ${ }^{1}$, Xiaofan $\mathrm{Li}^{2,3} \wedge$, Rong Liu ${ }^{1}$, Bijuan Lin ${ }^{4}$, Jingnan Xiang ${ }^{5}$, Wei Tian ${ }^{6}$, \\ Junjie Bai ${ }^{1}$, Teng Zuo ${ }^{1}$, Bingxin Lin ${ }^{1}$, Yinan Guo ${ }^{1}$, Song Zheng ${ }^{1}$ \\ ${ }^{1}$ Department of Urology, Fujian Medical University Union Hospital, Fuzhou, China; ${ }^{2}$ Department of Hematology, Fujian Institute of Hematology, \\ Union Hospital, Fujian Medical University, Fuzhou, China; ${ }^{3}$ Fujian Provincial Key Laboratory on Hematology, Fujian Medical University, Fuzhou, \\ China; ${ }^{4}$ Department of Pharmacy, Fujian Medical University Union Hospital, Fuzhou, China; ${ }^{5}$ Department of Emergency, Union Hospital, Fujian \\ Medical University, Fuzhou, China; ${ }^{6}$ Department of Dermatology, Fujian Medical University Union Hospital, Fuzhou, China \\ Contributions: (I) Conception and design: J Chen, S Zheng; (II) Administrative support: J Chen, S Zheng, W Jiang, B Lin, Y Guo; (III) Provision of \\ study materials or patients: J Chen, S Zheng, W Jiang; (IV) Collection and assembly of data: J Chen, W Ye, X Li, R Liu, J Xiang, W Tian, J Bai, T Zuo; \\ (V) Data analysis and interpretation: J Chen, W Ye, X Li, B Lin; (VI) Manuscript writing: All authors; (VII) Final approval of manuscript: All authors. \\ Correspondence to: Song Zheng. Department of Urology, Fujian Medical University Union Hospital, No. 29, Xinquan Road, Gulou District, Fuzhou \\ 350001, China. Email: zhengwu_99@aliyun.com.
}

Background: The efficacy and safety of pazopanib in patients diagnosed with metastatic renal cell carcinoma (mRCC) have been demonstrated by a Chinese subgroup analysis of the COMPARZ (Pazopanib Versus Sunitinib in the Treatment of Locally Advanced and/or Metastatic Renal Cell Carcinoma) trial. However, the real-world data are still unknown. This single-center, retrospective study was designed to verify the real-world effects of pazopanib in Chinese patients with mRCC.

Methods: Patients with mRCC and a clinical decision to initiate pazopanib as first-line therapy were eligible. The primary endpoint was progression-free survival (PFS), with overall survival (OS), objective response rate (ORR), and safety being evaluated as secondary endpoints. The effectiveness according to the International Metastatic Renal Cell Carcinoma Database Consortium (IMDC) risk model, number of risk factors in the intermediate risk group, age, Eastern Cooperative Oncology Group (ECOG) performance status (PS), and the number and site of organ metastasis were also assessed.

Results: A total of 32 patients were enrolled, including $23(71.9 \%)$ males and 9 (28.1\%) females. The median age was 57 years (range 29-75 years). With a median follow-up time of 23.8 months, a median PFS of 18.3 months, and an ORR of 37.5\%. Median OS was not reached, and the 1-, 2-, and 3-year overall survival rates were $90.6 \%, 78.1$, and $65.6 \%$, respectively. According to IMDC risk model, $37.5 \%$ were placed in the favorable risk (FR) subgroup, $56.2 \%$ (the majority) were placed in the intermediate risk (IR) subgroup, and $6.3 \%$ were placed in the poor risk (PR) subgroup. Compared with the IR and PR groups, the FR group achieved the best ORR (58.3\%) and median PFS (22.1 months). Having 1 risk factor, ECOG PS <2, 1 organ metastasis site, and only lung metastasis associated with a higher ORR and better median PFS. The IMDC risk model and number of metastases were associated with PFS. The most common adverse events were change in hair color $(69.0 \%)$, diarrhea (63\%), and hypertension $(50 \%)$.

Conclusions: Pazopanib showed efficacy and safety in real-world Chinese mRCC patients.

Keywords: Metastatic renal cell carcinoma (mRCC); pazopanib; real-world; the International Metastatic Renal Cell Carcinoma Database Consortium risk model (IMDC risk model)

Submitted Dec 23, 2020. Accepted for publication Mar 03, 2021.

doi: $10.21037 /$ tau-21-111

View this article at: http://dx.doi.org/10.21037/tau-21-111

^ORCID: Jianhui Chen, 0000-0001-7494-1258; Xiaofan Li, 0000-0002-6460-7210. 


\section{Introduction}

Renal cell carcinoma (RCC) accounts for the majority of kidney cancers (approximately 80-90\%), with nearly $75-80 \%$ of RCC patients having the clear cell histology type (1). In China, the incidence of RCC had increased from 3.96/100,000 in 2005 to 4.99/100,000 in 2014 (2). Metastatic RCC (mRCC) is one of the most treatmentresistant cancers; approximately $25-30 \%$ of RCC patients have distant metastasis at diagnosis, and $20-30 \%$ of RCC patients who undergo nephrectomy will develop mRCC (3). The widespread adoption of National Comprehensive Cancer Network (NCCN) guideline for RCC (4) was based on clinical trials mostly conducted in western population. Considering the global variability in patient characteristics and medical practice, there is an urgent need to clarify the management of mRCC specific to Chinese

Pazopanib, an oral receptor tyrosine kinase inhibitor (TKI), can inhibit vascular endothelial growth factor receptor (VEGFR), platelet-derived growth factor receptor (PDGFR) and c-Kit, exerting an antiangiogenesis effect that can inhibit both tumor cell proliferation and metastasis (5). The safety and efficacy of pazopanib in advanced metastatic RCC patients were demonstrated in a randomized, doubleblind, phase III trial (6). Furthermore, the COMPARZ (Pazopanib Versus Sunitinib in the Treatment of Locally Advanced and/or Metastatic Renal Cell Carcinoma) trial, a randomized, double-blind, sunitinib-controlled phase III study, demonstrated the noninferiority of pazopanib to sunitinib in respect to progression-free survival (PFS) and overall survival (OS), with the safety and quality-oflife profiles favoring pazopanib $(7,8)$. In a pooled analysis of a Chinese subgroup of the COMPARZ trial, pazopanib and sunitinib showed similar PFS. However, pazopanib demonstrated better safety in the Chinese subgroup (9). Therefore Pazopanib was recommended as first-line therapy for relapsed or stage IV surgically unresectable clearcell RCC cases in the National Comprehensive Cancer Network (NCCN) guidelines and the Chinese Society of Clinical Oncology (CSCO) guidelines with a high level of evidence $(4,10)$.

In China, pazopanib is now one of the most frequently used VEGFR-TKIs for mRCC patients. However, selecting the appropriate TKI for an individual patient can be challenging. Several real-world factors must be considered, including the experiences of urologists, the efficacy of TKIs, the different incidence rates of adverse events (AEs), patient symptoms, and Eastern Cooperative Oncology
Group (ECOG) performance status (PS) score. Due to this uncertainty, real-world studies have been used to complement randomized controlled trials (RCTs) by including more diverse patient populations, and improving the quantity and quality of the evidence used in guidelines (11).

Thus, as RCTs may not be representative of real-world practice, our study aimed to evaluate the efficacy and safety of first-line pazopanib in Chinese mRCC patients.

We present the following article in accordance with the STROBE reporting checklist (available at http://dx.doi. org/10.21037/tau-21-111).

\section{Methods}

\section{Study design and patient population}

This was a single-center, retrospective study conducted to determine the efficacy and safety of pazopanib therapy for mRCC. The study enrolled patients who were admitted to Fujian Medical University Union Hospital from 1st May 2017 to 31st January 2020. Data were retrieved from the electronic medical records and reviewed retrospectively. To be eligible, patients were required to meet the following inclusion criteria: (I) histologically confirmed advanced mRCC; (II) pazopanib received as first-line treatment according to the conventional schedule of $800 \mathrm{mg} /$ day; (III) aged $\geq 18$ years; last date of following 30th August 2020 . Pazopanib ( $800 \mathrm{mg} /$ day) was administered orally without interruption until disease progression, unacceptable toxicities, or mortality. Dose modification or discontinuation was administered according to the patients' tolerance. Clinical and laboratory data collected in the initiation of pazopanib treatment included International Metastatic Renal Cell Carcinoma Database Consortium (IMDC) risk (12), age, sex, ECOG PS score, histologic type, previous nephrectomy, previous local therapy, number and site of metastasis, and comorbidity. Ethical approval was obtained from the institutional ethics committee of Fujian Medical University Union Hospital (NO.: 2021KY011). The study was conducted in accordance with the Declaration of Helsinki (as revised in 2013), and written informed consent was provided by all patients prior to pazopanib treatment.

\section{Assessments}

The primary endpoint was PFS, defined as the time from pazopanib initiation to physician-assessed disease progression or death due to any cause, whichever occurred 
first. The secondary endpoint included objective response rate ORR that was defined as the proportion of patients with complete response (CR) or partial response (PR) according to Response Evaluation Criteria in Solid Tumors (RECIST) version 1.1. Other secondary endpoints were treatmentrelated adverse events (AEs) and OS. AEs assessed by common terminology criteria for adverse events (CTCAE) 4.0 version. OS was defined as the time from the starting of pazopanib to death for any cause. Prior to treatment, Patient demographics, medical history, physical examination and disease characteristics were recorded in detail as the baseline data. Efficacy was evaluated i approximately every 3 months ( \pm 4 weeks), and safety assessments were carried out every 4 weeks. Tumor responses were evaluated based on the radiologist's assessment and the physician's clinical judgment.

\section{Statistical analysis}

Continuous variables are expressed as medians and ranges, and categorical variables are expressed as numbers and percentages. Evaluation of data were based on $95 \%$ confidence intervals (CIs) and point estimates. Median PFS and ORR were stratified by baseline characteristics, including age, the number of metastatic organs, baseline bone, lung, and liver metastasis etc. Kaplan-Meier analysis was used to analyze PFS and OS. Comparisons between arms were made using a log-rank test (one sided) that was stratified by an IMDC risk subgroup. To find the impact of baseline characteristics on efficacy outcomes, Cox proportional hazards regression models (for PFS and OS) were used. All analyses were performed using SPSS version 19 (IBM Corp.). A P value $<0.05$ was considered statistically significant.

\section{Results}

\section{Patients and baseline demographics}

A total of 32 patients, with a median age of 57 years (range 29-75 years), were selected, 23 of whom were men; $78.1 \%$ patients had an ECOG PS $\geq 2$, and $71.9 \%$ patients were clear cell carcinoma. According to IMDC criteria, the percentages of patients with favorable risk (FR), intermediate risk (IR), and poor risk (PR) were $37.5 \%$, $56.2 \%$, and $6.3 \%$, respectively. The median number of metastatic organs was 1 (range 1-5), and patients with 1 metastatic organ accounted for $53 \%$. The remaining $47 \%$ of patients had 2 or more metastatic lesions, mostly involving the lungs and bone. Fifteen patients $(46.9 \%)$ had preexisting comorbidities, including hypertension, diabetes, cardio- and cerebrovascular disease, and immune-related disease. Hypertension $(46.9 \%)$ was the most common comorbid illness. Seventeen $(53.1 \%)$ patients received nephrectomy, with five of these patients experiencing cytoreductive nephrectomy. At the time of data collection, all patients were followed up. The median follow-up duration was 23.8 months [95\% confidence interval (CI), 6.5-41.6 months]. The patients' baseline demographics and clinical characteristics are summarized in Table 1.

\section{Clinical outcomes}

Median PFS was 18.3 months (95\% CI: 15.9-20.7 months; Figure $1 A$ ). At the time of analysis, 12 patients $(37.5 \%)$ were still in follow-up for progression. Of the all patients, objective progression was observed in 24 (75\%). Median OS has not yet been reached. The 1-, 2- and 3-year survival probabilities were $90.6 \%, 78.1 \%$, and $65.6 \%$, respectively. Twelve patients $(37.5 \%)$ died during the study, with the cause of death being the disease under study in 12 of 12 patients (100\%). No patients showed complete response (CR), 12 patients had partial response (PR), and 18 patients had stable disease (SD), resulting in an ORR of $37.5 \%$.

According to IMDC criteria, $12(37.5 \%)$ patients had 0 factors, 12 (37.5\%) had 1 factor, $6(18.8 \%)$ had 2 factors, 1 (3.1\%) had 3 factors, and 1 (3.1\%) had 4 factors. Thus, 12 were classified into the FR subgroup (37.5\%), 18 were classified into the IR subgroup (56.3\%), and 2 were classified into the PR subgroup (6.2\%). The ORR was the highest in the FR subgroup (58.3\%), compared with IR subgroup and PR subgroup (Table 2). Median PFS showed a statistically significant difference among the subgroups (22.1 months in the FR subgroup, 17.1 months in the IR subgroup, and 5 months in the PR subgroup; $\mathrm{P}<0.001$; Figure $1 B$ ). For the percentage of maximal tumor change, 30 tumors were downsized (Figure 2). Partial response was observed in 7 tumors (58.3\%) in the FR subgroup, 5 (27.8\%) tumors in the IR subgroup, and 0 tumors in PR subgroup.

The subgroup analyses are presented in Table 3. In the IR subgroup, median PFS was 17.8 months (95\% CI: $15.7-$ 19.9 months) and 8.0 months (95\% CI: 3.3-12.9 months) for patients with 1 and 2 risk factors, respectively (Table 3). This means that patients who had 1 risk factor had longer median PFS. In the ECOG PS analysis, a higher ORR and median PFS were found for patients with PS $<2$ as 
Table 1 Baseline patient characteristics

\begin{tabular}{|c|c|}
\hline Variables & No. of patients (\%) \\
\hline \multicolumn{2}{|l|}{ Age, years } \\
\hline Median [range] & 57 [29-75] \\
\hline \multicolumn{2}{|l|}{ Sex } \\
\hline Female & $9(28.1)$ \\
\hline Male & $23(71.9)$ \\
\hline \multicolumn{2}{|c|}{ ECOG performance status score } \\
\hline$<2$ & $27(84.4)$ \\
\hline$\geq 2$ & $5(15.6)$ \\
\hline \multicolumn{2}{|l|}{ Histology } \\
\hline Clear cell carcinoma & $22(68.8)$ \\
\hline Non-clear cell carcinoma & $10(31.2)$ \\
\hline Type I papillary & $3(9.4)$ \\
\hline Type II papillary & $2(6.3)$ \\
\hline Sarcomatoid variant & $2(6.3)$ \\
\hline Xp11.2 translocation & $2(6.3)$ \\
\hline Collecting duct & $1(3.1)$ \\
\hline \multicolumn{2}{|l|}{ IMDC risk } \\
\hline Favorable risk & $12(37.5)$ \\
\hline Intermediate risk & $18(56.25)$ \\
\hline Poor risk & $2(6.3)$ \\
\hline \multicolumn{2}{|l|}{ Number of metastatic organs } \\
\hline Median [range] & $1[1-5]$ \\
\hline \multicolumn{2}{|l|}{ Sites of metastatic organ } \\
\hline Lung & $16(50.0)$ \\
\hline Bone & $9(28.1)$ \\
\hline Lymph node & 7 (21.9) \\
\hline Liver & $6(18.8)$ \\
\hline Other & $6(18.8)$ \\
\hline \multicolumn{2}{|l|}{ Number of metastasis sites } \\
\hline 1 & $17(53.1)$ \\
\hline$\geq 2$ & $15(46.9)$ \\
\hline
\end{tabular}

Table 1 (continued)
Table 1 (continued)

\begin{tabular}{lc}
\hline Variables & No. of patients (\%) \\
\hline Comorbidities & $15(46.9)$ \\
Yes & $15(46.9)$ \\
Hypertension & $3(9.4)$ \\
Diabetes & $4(12.5)$ \\
Cardio- and cerebrovascular diseases & $1(3.1)$ \\
Immune-related diseases & $17(53.1)$ \\
Prior nephrectomy & $15(46.9)$ \\
Yes & \\
No & $5(15.6)$ \\
Local therapy & $5(15.6)$ \\
Cytoreductive nephrectomy & $5(15.6)$ \\
\hline Metastasectomy & \\
SBRT for oligometastatic sites & \\
\hline ECOG, Eastern Cooperative Oncology Group; IMDC, International \\
Metastatic Renal Cell Carcinoma Database Consortium; SBRT, \\
stereotactic body radiation therapy.
\end{tabular}

compared to those with a PS $\geq 2$. For ECOG PS $<2$, the ORR was $48 \%$, and the median PFS was 20.1 months, compared to $0 \%$ and 8.1 months for patients with ECOG PS $\geq 2$ (Table 3). Meanwhile, for the number of metastatic sites, patients with $<1$ metastasis site showed a higher ORR (64.7\%) and median PFS (23.7 months) (Table 3). Finally, in the metastasis site analysis, patients with solitary lung metastasis showed the highest ORR (66.7\%) and median PFS (22.1 months) (Table 3).

\section{Prognostic factors for PFS}

We used the univariable and multivariable Cox regression to analyze the prognostic factors for PFS among mRCC patients treated with pazopanib (Table 4). Univariate analyses identified 3 significant prognostic predictors for PFS, including prior nephrectomy, number of metastases, 
A

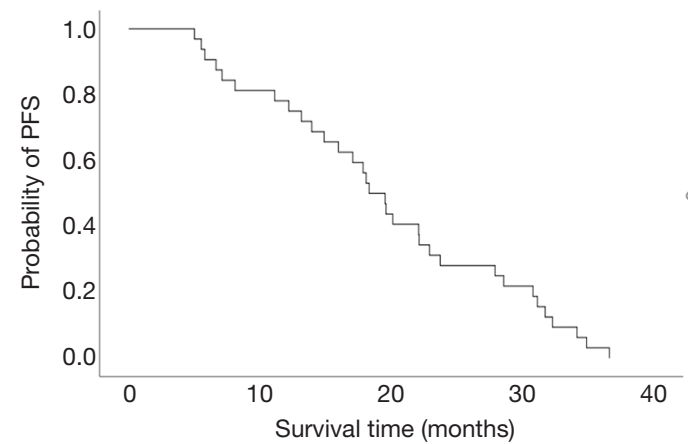

B

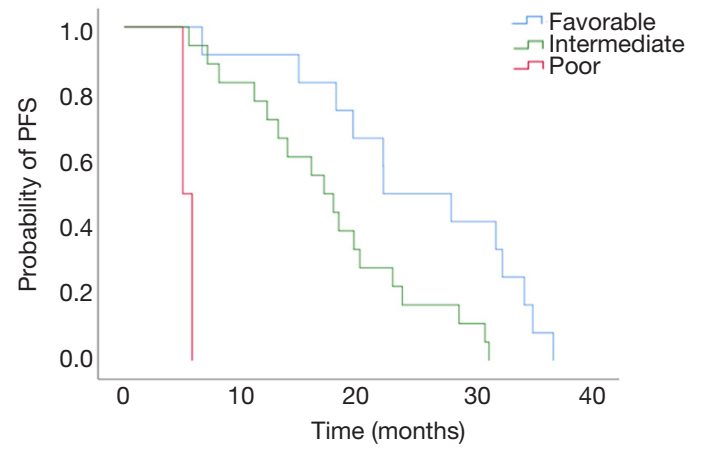

Figure 1 Progression-free survival analysis. Progression-free survival in the total population (A) and in the IMDC risk subgroups (B). IMDC, International Metastatic Renal Cell Carcinoma Database Consortium.

Table 2 Response rates for pazopanib treatment in the total population and according to IMDC risk groups

\begin{tabular}{lcccc}
\hline Variable & Total population, $\mathrm{n}(\%)$ & Favorable risk, $\mathrm{n}(\%)$ & Intermediate risk, $\mathrm{n}(\%)$ & Poor risk, $\mathrm{n}(\%)$ \\
\hline $\mathrm{N}$ & 32 & 12 & 18 & 2 \\
Complete response & $0(0)$ & $0(0)$ & $0(0)$ & $0(0)$ \\
Partial response & $12(37.5)$ & $7(58.3)$ & $5(27.8)$ & $0(0)$ \\
Stable disease & $18(56.3)$ & $5(41.7)$ & $12(66.7)$ & $1(50.0)$ \\
Partial disease & $2(18.1)$ & $0(0)$ & $1(5.5)$ & $1(50.0)$ \\
Objective response rate & $12(37.5)$ & $7(58.3)$ & $5(27.8)$ & $0(0)$ \\
Disease control rate & $29(90.6)$ & $12(100)$ & $17(94.4)$ & $1(50.0)$ \\
\hline
\end{tabular}

IMDC, International Metastatic Renal Cell Carcinoma Database Consortium.

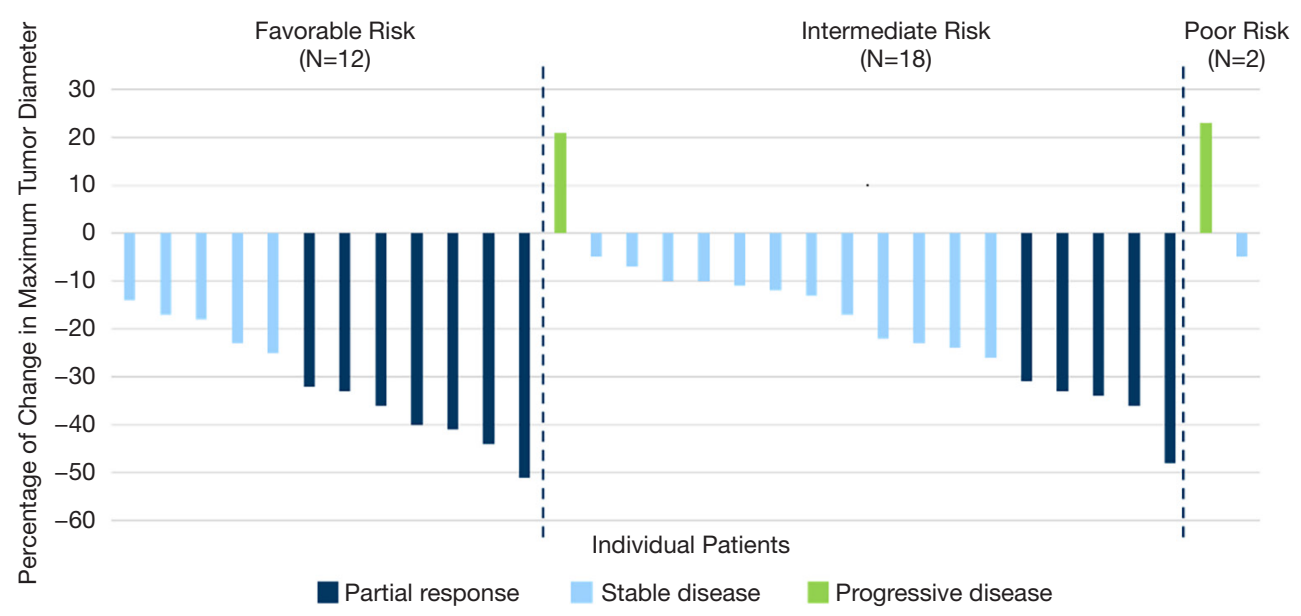

Figure 2 The maximum percent change in primary tumor diameter following pazopanib according to different IMDC risk stages. Each bar represents 1 of 32 individual patients. IMDC, International Metastatic Renal Cell Carcinoma Database Consortium. 
Table 3 Efficacy in patient subgroups analysis

\begin{tabular}{|c|c|c|c|}
\hline Subgroup & \multicolumn{2}{|r|}{ PFS } & ORR, n/N (\% \\
\hline \multicolumn{4}{|l|}{ Age, y } \\
\hline$<65$ & $15 / 22$ & $18.8(14.9-23.2)$ & 8/22 (36.4) \\
\hline$\geq 65$ & $9 / 10$ & $18.3(13.4-28.1)$ & $4 / 10(40.0)$ \\
\hline$<2$ & $18 / 25$ & $20.1(15.5-30.6)$ & $12 / 25(48)$ \\
\hline$\geq 2$ & $6 / 7$ & $8.1(5.0-15.6)$ & $0 / 7(0)$ \\
\hline \multicolumn{4}{|l|}{ Number of metastatic organs } \\
\hline 1 & $10 / 17$ & $23.7(20.6-29.2)$ & $11 / 17(64.7)$ \\
\hline 1 & $9 / 12$ & $20.4(15.7-23.8)$ & $5 / 12(41.7)$ \\
\hline 2 & $6 / 6$ & $8.3(3.9-22.7)$ & $0 / 6(0)$ \\
\hline \multicolumn{4}{|l|}{ Site of organ metastasis } \\
\hline Lung metastasis & $12 / 17$ & $18.3(13.9-22.7)$ & 6/17 (35.3) \\
\hline Solitary lung metastasis & $4 / 9$ & $22.1(14.9-27.8)$ & $6 / 9(66.7)$ \\
\hline Bone metastasis & $9 / 9$ & $8.0(5.3-19.4)$ & $0 / 9(0)$ \\
\hline Liver metastasis & $6 / 6$ & $11.6(4.0-24.2)$ & $0 / 6(0)$ \\
\hline
\end{tabular}

PFS, progression-free survival; ORR, objective response rate; ECOG PS, Eastern Cooperative Oncology Group performance status.

and bone metastasis. The results of multivariate analysis suggested that a shorter PFS was strongly associated with the following prognostic factors: $>1$ metastatic organ and PR.

\section{Safety}

Adverse events were reported in 30 patients (93.8\%), the majority of which were Common Terminology Criteria for Adverse Events (CTCAE) grade 1 or 2 in severity. The 3 most common treatment-related AEs were change in hair color $(69.0 \%)$, diarrhea (63.0\%), and hypertension (50.0\%). Change in hair color (69\%), diarrhea (47\%), fatigue (44\%), and stomatitis (38\%) were the most common grade 1-2 AEs. The 4 most common treatment-related grade 3 events were diarrhea $(16.0 \%)$, hypertension $(16.0 \%)$, hand-foot syndrome $(16.0 \%)$, and increased alanine aminotransferase (ALT) (12.0\%). No grade 4 toxicity was reported. In all, 32 patients $(100 \%)$ started first-line pazopanib at $800 \mathrm{mg}$ daily, and $16(50 \%)$ of these patients required dose reductions due to AEs. The treatment-related AEs that occurred in at least $10 \%$ of patients are summarized in Table 5.

\section{Discussion}

Pazopanib is the first-line TKI treatment of mRCC patients and exerts an antiangiogenic effect that inhibits cancer cell proliferation and metastasis $(5,13)$. The COMPARZ study demonstrated the non-inferiority of pazopanib versus sunitinib in PFS and OS on the basis of independent review committee (IRC) evaluation $(7,8)$. According to the investigator assessment of the Chinese population analysis of the COMPARZ trial, no significant difference in median PFS was found between the two therapies, with 13.9 months for pazopanib and 14.3 months for sunitinib. Patients treated with sunitinib had a median OS of 29.5 months until followup, whereas median OS has not been reached for pazopanib. A significantly higher ORR was observed in the pazopanib group (investigator assessment: $41 \%$ versus $23 \%, \mathrm{P}=0.0052$; independent review: $35 \%$ versus $20 \%, \mathrm{P}=0.0203$ ) (9). This result indicated that pazopanib has a better tumor-shrinking 
Table 4 Prognostic factors for PFS among patients treated with pazopanib

\begin{tabular}{|c|c|c|}
\hline Variable & $\begin{array}{l}\text { Univariate } \\
\text { ( } P \text { value) }\end{array}$ & $\begin{array}{c}\text { Multivariate } \\
\text { ( } P \text { value) }\end{array}$ \\
\hline \multicolumn{3}{|l|}{ Sex } \\
\hline Male/female & 0.7 & - \\
\hline \multicolumn{3}{|l|}{ Age } \\
\hline$<65 / \geq 65$ & 0.6 & - \\
\hline \multicolumn{3}{|l|}{ Histology } \\
\hline ccRCC/non-ccRCC & 0.1 & - \\
\hline \multicolumn{3}{|l|}{ ECOG performance status } \\
\hline$<2 / \geq 2$ & 0.017 & 0.724 \\
\hline \multicolumn{3}{|l|}{ Prior nephrectomy } \\
\hline Yes/no & 0.001 & 0.064 \\
\hline \multicolumn{3}{|l|}{ Local therapy } \\
\hline Yes/no & 0.09 & - \\
\hline \multicolumn{3}{|l|}{ Number of metastatic organs } \\
\hline $1 />1$ & 0.001 & 0.005 \\
\hline \multicolumn{3}{|l|}{ Lung metastasis } \\
\hline Yes/no & 0.3 & - \\
\hline \multicolumn{3}{|l|}{ Bone metastasis } \\
\hline Yes/no & 0.002 & - \\
\hline \multicolumn{3}{|l|}{ IMDC risk subgroup } \\
\hline Favorable/intermediate/poor & - & 0.04 \\
\hline
\end{tabular}

PFS, progression-free survival; ccRCC, clear cell renal cell carcinoma; non-ccRCC, non-clear cell renal cell carcinoma; ECOG, Eastern Cooperative Oncology Group; IMDC, International Metastatic Renal Cell Carcinoma Database Consortium.

effect than sunitinib in the Chinese population. However, there remains a need to validate the effectiveness in a realworld Chinses population outside of clinical trials. This retrospective study found that in first-line real-world Chinese mRCC patients, treatment with pazopanib was both well tolerated and effective. Pazopanib achieved an objective response in $37.5 \%$ of patients. The estimated median PFS was 18.3 months (95\% CI: 15.9-20.7 months). These results compared favorably with the outcomes of the Chinese subgroup pooled analysis of the COMPARZ study, and indicate that Chinese patients may derive clinical benefits from pazopanib treatment.

The treatment of mRCC has entered an era where
Table 5 Treatment-related adverse events occurring in at least $10 \%$ of patients $(\mathrm{n}=32)$

\begin{tabular}{|c|c|c|c|}
\hline Adverse events & $\begin{array}{c}\text { No. grade } \\
1 / 2[\%]\end{array}$ & $\begin{array}{c}\text { No. grade } \\
3[\%]\end{array}$ & $\begin{array}{c}\text { Total No. } \\
{[\%]}\end{array}$ \\
\hline $\begin{array}{l}\text { Changes in hair } \\
\text { color }\end{array}$ & 22 [69] & $0[0]$ & 22 [69] \\
\hline Diarrhea & $15[47]$ & $5[16]$ & 20 [63] \\
\hline Hypertension & $11[34]$ & $5[16]$ & $16[50]$ \\
\hline Fatigue & 14 [44] & $1[3]$ & $15[47]$ \\
\hline Nausea & $13[41]$ & $0[0]$ & $13[41]$ \\
\hline $\begin{array}{l}\text { Hand-foot } \\
\text { syndrome }\end{array}$ & 7 [22] & $5[16]$ & $12[38]$ \\
\hline Stomatitis & 12 [38] & $0[0]$ & $12[38]$ \\
\hline Increased ALT & 7 [22] & $4[12]$ & $11[34]$ \\
\hline Increased AST & 7 [22] & $3[9]$ & $10[31]$ \\
\hline Alopecia & 9 [28] & $0[0]$ & 9 [28] \\
\hline Vomit & 8 [25] & $1[3]$ & 9 [28] \\
\hline Abdominal pain & 8 [25] & $0[0]$ & 8 [25] \\
\hline Neutrocytopenia & $5[16]$ & $2[6]$ & 7 [22] \\
\hline Rash & $5[16]$ & $1[3]$ & 6 [19] \\
\hline Proteinuria & $3[10]$ & $3[10]$ & 6 [19] \\
\hline hypothyroidism & $5[16]$ & $0[0]$ & 5 [16] \\
\hline $\begin{array}{l}\text { Hemoglobin } \\
\text { decreased }\end{array}$ & $4[13]$ & $0[0]$ & 4 [13] \\
\hline
\end{tabular}

ALT, alanine aminotransferase; AST, aspartate aminotransferase.

immunotherapy and targeted therapy coexist. The NCCN guidelines have recommended three immune combination therapies (nivolumab + ipilimumab, pembrolizumab + axitinib, avelumab + axitinib) and five targeted drugs (pazopanib, sunitinib, axitinib, cabozantinib, temsirolimus) as the first-line treatment (14). However, there is a lack of reliable biomarkers to guide oncologists in choosing between immunotherapy and targeted drugs. The IMDC model was still used externally to stratify mRCC patients in clinical trials and to guide mRCC patient prognosis (15). At 42 months of follow-up in the CheckMate 214 study, nivolumab plus ipilimumab demonstrated improved efficacy outcomes versus sunitinib in IR and PR patients. However, in FR patients, the ORR was $54.0 \%$ and $28.8 \%$ for sunitinib versus nivolumab + ipilimumab, respectively, while the median PFS was 28.8 and 17.0 months, respectively (16). This means that in FR patients, both ORR and median 
PFS can be improved with TKIs alone. In our real-world study, FR patients achieved a high ORR (58.3\%) and longer median PFS (22.1 months) with pazopanib, compared with the IR and PR patients.

Based on Keynote 426 study, the survival benefit of pembrolizumab + axitinib was observed across all of the IMDC risk groups (17). Therefore the combination of pembrolizumab + axitinib was recommended as first-line treatment in the NCCN guidelines (14). However, in the 2020 updated analysis of Keynote-426, in FR patients, the 2 -years OS rate was $85.3 \%$ and $87.7 \%$ for pembrolizumab + axitinib versus sunitinib (HR $=1.06,95 \%$ CI, 0.60-1.86, $\mathrm{P}=0.58)$, respectively, the median PFS was 20.8 and 18.0 months (HR $=0.79,95 \%$ CI, 0.51-1.09, $\mathrm{P}=0.078)$ (18). So in FR patients, the survival benefit of combination therapy versus sunitinib is not as obvious as in IR and PR patients. Treatment strategies for advanced renal cell carcinoma should focus on the entire treatment process. However, there is no clinical trial similar to SWICH and SWITH II study $(19,20)$, which was designed to compare the overall survival of mRCC with the strategies of first-line immunotherapy followed by TKIs or first-line TKIs with subsequent immunotherapy. Furthermore, from the perspective of pharmacoeconomics, pembrolizumab + axitinib is not costeffective when compared with sunitinib for mRCC patients as first-line therapy in China (21). Even in the United States, it is controversial whether the cost-effectiveness of combination therapy is superior to sunitinib for mRCC patients, especially for FR patients $(22,23)$. Currently, in China, pazopanib has been included in the medical insurance and charity policy, and its treatment costs are similar to or even less than sunitinib. From this cost-effectiveness point of view, pazopanib may be the first-line treatment for patients with low-risk advanced renal cell carcinoma.

Apart from favorable risk, it is unclear whether all patients with intermediate risk should be treated by the same strategy, as the IR patient group in our study comprised 1 or 2 risk factors. The PRICINPAL (Prospective Observational Study of Pazopanib in Patients with Advanced Renal Cell Carcinoma) study, a large prospective real-world study, demonstrated that intermediate risk patients with 1 risk factor had a longer median PFS than those with 2 factors (24). In our study, we found that the median PFS was 17.8 months for those with 1 risk factor versus 8.0 months for those with 2 risk factors in the Chinese population. This result was in line with previous retrospective study, in which patients with 1 risk factor received longer PFS than those with 2 risk factors (25-27).
In a Korean real-world study, complete response and partial response patients had favorable risk, a longer disease-free interval to systemic therapy, a greater proportion of lung metastasis, and less metastasis, compared with stable disease and progressive disease patients (28). Our clinical subgroup analysis outcomes also exhibited similar trends, including number of metastatic organs, site of metastatic organs, and ECOG PS. For PR and long median PFS patients in our study, we observed a greater proportion of patients with ECOG PS $<2$, more than 1 metastatic organ, and only lung metastasis. Our findings suggest that the intermediate prognostic risk model can be further stratified into Chinese mRCC patients, a subgroup composed of factor number, ECOG PS $<2,1$ metastatic, organ, and only lung metastasis. This may lead to the greater prediction accuracy of pazopanib treatment outcomes. Our findings may increase the suitability of treatment choices for Chinese mRCC patients, particularly if applied to the real-world treatment of programmed cell death protein 1 (PD-1) combined with TKI vs. TKI therapy. Of course, based on the CheckMate 016 study, pazopanib in combination with pembrolizumab occurred unacceptable toxicity (29). So in the real world, we should try to avoid using pazopanib in combination with pembrolizumab. If this protocol is chosen, pazopanib must also be reduced to $600 \mathrm{mg} /$ day or less for combination therapy, with attention to monitoring for AEs such as druginduced hepatotoxicity. Despite the superior efficacy found with the combination of TKI and immunotherapy, the use of pazopanib monotherapy is still a viable option for $\mathrm{mRCC}$ patients, particularly in these subsets: patients with 1 risk factor, ECOG PS <2, 1 metastatic, organ, and only lung metastasis.

In addition to the above, our results found that that high tumor burden (>1 metastatic organ) and poor-risk were negative prognostic factors for PFS in mRCC patients, which consistent with previous pazopanib studies $(28,30)$.

Overall, the safety of pazopanib observed in this study was similar to that observed in the COMPARZ Chinese subgroup pooled study (9). Diarrhea and hypertension were the most common AEs. The most concerning AE of increased alanine aminotransferase/aspartate transaminase was mainly grade $1-2$, and cases of grade 3-4 AEs occurred less frequently than in other studies, including the COMPARZ trial. Compared with COMPARZ Chinese subgroup pooled analysis, bone marrow suppression and hepatotoxicity occurred in fewer cases, while diarrhea, hypertension, nausea, and hair color changes appeared more frequently. The AE profile was acceptable. Because the 
AE profile of pazopanib was different from sunitinib, the severity of hematologic toxicity and the hand-foot syndrome was mild, and the incident rate was lower than that of sunitinib. Therefore, pazopanib is suitable for patients with grade 3 or above hematologic $\mathrm{AE}$ and intolerability of hand-foot syndrome during sunitinib treatment (30-32). In a randomized, double-blind, crossover, controlled, phase III study to compare the quality of life (QOL) and drug safety of patients treated with pazopanib versus sunitinib (PISCES study), The primary endpoint was patient preference, assessed at 22 weeks. When asked about reasons for selecting one drug over another, about $70 \%$ selected pazopanib due to better QOL, compared with $22 \%$ of the sunitinib-treated patients and the remaining $8 \%$ of patients having no preference (33). Therefore, pazopanib could be a better first-line TKI for mRCC than sunitinib.

In our study, the median PFS reach to 18.3 months, longer than most reported studies $(6,8,9,30)$. The following reasons may explain. There is a potential selection bias to this retrospective study. According to the investigator assessment of the Chinese population subgroup analysis of the COMPARZ trial, favorable-risk, intermediate-risk, and Poor-risk patients represented 22\%, 64\%, and $13 \%$, respectively, of the pazopanib cohort (9). In our study, the composition ratio was $37.5 \%, 56.2 \%$, and $6.3 \%$. It follows that our study had a lower proportion of poorrisk patients with poor clinical outcomes compared to the COMPARZ trial. In addition, our single-center data showed that more than half of the patients in this study had previous renal resection, and $40 \%$ of the patients received local cytoreductive treatment (primary tumor resection, metastasis tumor resection, or stereotactic body radiation therapy). RCC is a highly heterogeneous malignant tumor. Theoretically, TKI treatment alone cannot inhibit multiple tumor cells with different mutation pathways, which will eventually lead to resistance to systemic therapy. On the other hand, cytoreductive surgery and other treatments reduce the tumor burden, objectively minimize drugresistant tumor cell clones, delay the time of tumor resistance, and ultimately extend the effective time of therapy.

This study also had several limitations, including its small sample size, single-center data, short follow-up time, and retrospective design. However, our data were consistent and comparable with previous results reported in both randomized clinical trials and real-world studies. To more conclusively assess the real-world efficacy and safety of pazopanib in Chinese patients, large-sample, multicenter, prospective studies are needed. This way, a more precise evaluation and screening of the patient population can be realized.

\section{Acknowledgments}

The inclusion of co-authors reflects the fact that the work came from active collaboration between researchers and acknowledges input into team-based research.

Funding: This work was supported by grants from Natural Science Foundation of Fujian Province (2019J01153), and Startup Fund for scientific research, Fujian Medical University (2019QH1053).

\section{Footnote}

Reporting Checklist: The authors have completed the STROBE reporting checklist. Available at http://dx.doi. org/10.21037/tau-21-111

Data Sharing Statement: Available at http://dx.doi. org/10.21037/tau-21-111

Conflicts of Interest: All authors have completed the ICMJE uniform disclosure form (available at http://dx.doi. org/10.21037/tau-21-111). The authors have no conflicts of interest to declare.

Ethical Statement: The authors are accountable for all aspects of the work in ensuring that questions related to the accuracy or integrity of any part of the work are appropriately investigated and resolved. Ethical approval was obtained from the institutional ethics committee of Fujian Medical University Union Hospital (No. 2021KY011). The study was conducted in accordance with the Declaration of Helsinki (as revised in 2013), and written informed consent was provided by all patients prior to pazopanib treatment.

Open Access Statement: This is an Open Access article distributed in accordance with the Creative Commons Attribution-NonCommercial-NoDerivs 4.0 International License (CC BY-NC-ND 4.0), which permits the noncommercial replication and distribution of the article with the strict proviso that no changes or edits are made and the original work is properly cited (including links to both the formal publication through the relevant DOI and the license). See: https://creativecommons.org/licenses/by-nc-nd/4.0/. 


\section{References}

1. Deveson Kell S. Renal cell carcinoma: treatment options. Br J Nurs 2011;20:536-8.

2. China NHCotPsRo. Chinese guidelines for diagnosis and treatment of renal cell carcinoma 2018 (English version). Chin J Cancer Res 2019;31:29-48.

3. Gupta K, Miller JD, Li JZ, et al. Epidemiologic and socioeconomic burden of metastatic renal cell carcinoma (mRCC): a literature review. Cancer Treat Rev 2008;34:193-205.

4. Motzer RJ, Jonasch E, Agarwal N. Kidney Cancer, Version 2.2017, NCCN Clinical Practice Guidelines in Oncology. J Natl Compr Canc Netw 2017;15:804-34.

5. Pick AM, Nystrom KK. Pazopanib for the Treatment of Metastatic Renal Cell Carcinoma. Clin Ther 2012;34:511-20.

6. Sternberg CN, Davis ID, Mardiak J, et al. Pazopanib in locally advanced or metastatic renal cell carcinoma: results of a randomized phase III trial. J Clin Oncol 2010;28:1061-8.

7. Motzer RJ, Hutson TE, McCann L, et al. Overall Survival in Renal-Cell Carcinoma with Pazopanib versus Sunitinib. N Engl J Med 2014;370:1769-70.

8. Motzer RJ, Hutson TE, Cella D, et al. Pazopanib versus sunitinib in metastatic renal-cell carcinoma. $\mathrm{N}$ Engl J Med 2013;369:722-31.

9. Sheng X, Jin J, He Z, et al. Pazopanib versus sunitinib in Chinese patients with locally advanced or metastatic renal cell carcinoma: pooled subgroup analysis from the randomized, COMPARZ studies. BMC Cancer 2020;20:219.

10. Guo J, Ma J, Sun Y, et al. Chinese guidelines on the management of renal cell carcinoma (2015 edition). Ann Transl Med 2015;3:279.

11. Oyinlola JO, Campbell J, Kousoulis AA. Is real world evidence influencing practice? A systematic review of CPRD research in NICE guidances. BMC Health Serv Res 2016;16:299.

12. Heng DY, Xie W, Regan MM, et al. Prognostic factors for overall survival in patients with metastatic renal cell carcinoma treated with vascular endothelial growth factortargeted agents: results from a large, multicenter study. J Clin Oncol 2009;27:5794-9.

13. Hurwitz HI, Dowlati A, Saini S, et al. Phase I Trial of Pazopanib in Patients with Advanced Cancer. Clin Cancer Res 2009;15:4220-7.

14. Motzer RJ, Jonasch E, Michaelson MD, et al. NCCN
Guidelines Insights: Kidney Cancer, Version 2.2020. JNCCN 2019;17:1278-85.

15. Heng DY, Xie W, Regan MM, et al. External validation and comparison with other models of the International Metastatic Renal-Cell Carcinoma Database Consortium prognostic model: a population-based study. Lancet Oncol 2013;14:141-8.

16. Motzer RJ, Escudier B, McDermott DF, et al. Survival outcomes and independent response assessment with nivolumab plus ipilimumab versus sunitinib in patients with advanced renal cell carcinoma: 42-month follow-up of a randomized phase 3 clinical trial. J Immunother Cancer 2020;8:e000891.

17. Rini BI, Plimack ER, Stus V, et al. Pembrolizumab plus Axitinib versus Sunitinib for Advanced Renal-Cell Carcinoma. N Engl J Med 2019;380:1116-27.

18. Powles T, Plimack ER, Soulières D, et al. Pembrolizumab plus axitinib versus sunitinib monotherapy as firstline treatment of advanced renal cell carcinoma (KEYNOTE-426): extended follow-up from a randomised, open-label, phase 3 trial. Lancet Oncol 2020;21:1563-73.

19. Eichelberg C, Vervenne WL, De Santis M, et al. SWITCH: A Randomised, Sequential, Open-label Study to Evaluate the Efficacy and Safety of Sorafenib-sunitinib Versus Sunitinib-sorafenib in the Treatment of Metastatic Renal Cell Cancer. Eur Urol 2015;68:837-47.

20. Retz M, Bedke J, Bgemann M, et al. SWITCH II: Phase III randomized, sequential, open-label study to evaluate the efficacy and safety of sorafenib-pazopanib versus pazopanib-sorafenib in the treatment of advanced or metastatic renal cell carcinoma (AUO AN 33/11). Eur J Cancer 2019;107:37-45.

21. Chen J, Hu G, Chen Z, et al. Cost-effectiveness Analysis of Pembrolizumab Plus Axitinib Versus Sunitinib in Firstline Advanced Renal Cell Carcinoma in China. Clin Drug Investig 2019;39:931-8.

22. Ding D, Hu H, Shi Y, et al. Cost-Effectiveness of Pembrolizumab plus Axitinib Versus Sunitinib as FirstLine Therapy in Advanced Renal Cell Carcinoma in the U.S. Oncologist 2021;26:e290-7.

23. Zhu J, Zhang T, Wan N, et al. Cost-effectiveness of pembrolizumab plus axitinib as first-line therapy for advanced renal cell carcinoma. Immunotherapy 2020;12:1237-46.

24. Procopio G, Bamias A, Schmidinger M, et al. Realworld Effectiveness and Safety of Pazopanib in Patients With Intermediate Prognostic Risk Advanced Renal Cell Carcinoma. Clin Genitourin Cancer 2019;17:e526-33. 
25. Tamada S, Iguchi T, Yasuda S, et al. The difference in the survival rate of patients with metastatic renal cell carcinoma in the intermediate-risk group of the Memorial Sloan Kettering Cancer Center criteria. Oncotarget 2018;9:27752-9.

26. Sella A, Michaelson MD, Matczak E, et al. Heterogeneity of Patients With Intermediate-Prognosis Metastatic Renal Cell Carcinoma Treated With Sunitinib. Clin Genitourin Cancer 2017;15:291-9.e1.

27. Iacovelli R, De Giorgi U, Galli L, et al. Is it possible to improve prognostic classification in patients affected by metastatic renal cell carcinoma with an intermediate or poor prognosis? Clin Genitourin Cancer 2018;16:355-9.e1.

28. Kim MS, Chung HS, Hwang EC, et al. Efficacy of FirstLine Targeted Therapy in Real-World Korean Patients with Metastatic Renal Cell Carcinoma: Focus on Sunitinib and Pazopanib. J Korean Med Sci 2018;33:e325.

29. Amin A, Plimack ER, Ernstoff MS, et al. Safety and efficacy of nivolumab in combination with sunitinib or pazopanib in advanced or metastatic renal cell carcinoma:

Cite this article as: Chen J, Ye W, Jiang W, Li X, Liu R, Lin B, Xiang J, Tian W, Bai J, Zuo T, Lin B, Guo Y, Zheng S. Pazopanib in patients with metastatic renal cell carcinoma: a single-center, real-world, retrospective Chinese study. Transl Androl Urol 2021;10(3):1321-1331. doi: 10.21037/tau-21-111
The CheckMate 016 study. J Immunother Cancer 2018;6:109.

30. Schmidinger M, Bamias A, Procopio G, et al. Prospective Observational Study of Pazopanib in Patients with Advanced Renal Cell Carcinoma (PRINCIPAL Study). Oncologist 2019;24:491-7.

31. He Z, Guo G, Zhang C, et al. Efficacy of sunitinib in patients with metastatic renal cell carcinoma: initial experience in two Chinese centers. Chin Med J 2014;127:1450-3.

32. Yoo C, Kim JE, Lee J-L, et al. The efficacy and safety of sunitinib in korean patients with advanced renal cell carcinoma: high incidence of toxicity leads to frequent dose reduction. Jpn J Clin Oncol 2010;40:980-5.

33. Escudier B, Porta C, Bono P, et al. Randomized, controlled, double-blind, cross-over trial assessing treatment preference for pazopanib versus sunitinib in patients with metastatic renal cell carcinoma: PISCES Study. J Clin Oncol 2014;32:1412-8.

(English Language Editor: J. Gray) 